\title{
IMPACT OF DEMOGRAPHIC PROCESSES \\ ON REGIONAL ECONOMIC DEVELOPMENT
}

DOI: http://dx.doi.org/10.18509/GBP.2018.24

UDC: 332.14/.15:314(100)

\author{
Metodi Ivanov \\ Sofia University „St. Kliment Ohridski“, Sofia, Bulgaria
}

\begin{abstract}
With this report will attempt to present the impact of demographic processes on socioeconomic development in the regions. Given that globalization processes cover, besides the economy and almost all major spheres of public activity, such as politics, ideology, culture, way of life, and the conditions for the existence of mankind. A basic prerequisite for the development of the processes of globalization is the information revolution, which simultaneously creates and provides the technical basis for the development of global information networks, the internationalization of capital, as well as enhancing the competitive struggle on the world markets. At the heart of the demographic behavior of humans as well as of all living beings is the general biological law of the struggle for survival and continuation of the genus, which also determines the major trends in the world. This fact determines the migration of labor resources at the international level as wages in developed countries exceeds what workers receive with the same qualifications in less developed countries. In the age of globalization, migration policy is increasingly spontaneous and increasingly systematic and diversified. We must not overlook the fact that migration affects national employment, the quality of human capital, labor productivity, income levels, and even the state of interethnic relations. And in this line of thought, the current modern migration policy should include not only the direct regulation of migratory flows, but also the definition and implementation of measures for their integration into national society, economy and culture.
\end{abstract}

Keywords: demography, regional economy, regional development, migration

\section{INTRODUCTION}

Significant influence in the development of individual countries and regions is the change in demographic processes that affect long-lasting and sustainable trends in the socio-economic development of individual territorial units. Substantial impact on socioeconomic processes is the aging of the population, with the result that major challenges in aging countries and regions are related to health, social security, education, employment and income, housing, transport, the environment. The process of aging affects the reduction of the reproduction and deepening of the unevenness of age structure, as a result of which unfavorable demographic development is established. Reducing the mortality and birth rate of the world population is related to the parallel development of mankind, to the industrialization and economic progress of the individual countries and consequently to the raising of the living standards of the population. The demographic transition is mainly associated with most developed countries in Europe, North America and Japan, as their populations have gone through several phases of this process. The first major phase in the demographic transition is the antiquated phase, 
characterized by high birth rates and mortality, resulting in a relatively balanced demographic process characterized by very low natural growth. At this stage, the two processes were mutually neutralized, and thousands of years were needed to increase the absolute population. The next second phase is associated with the entry of industrialization, characterized by a steady decline in mortality, while birth rates remain at their relative levels. During this phase, the increase in life expectancy, as well as the decrease in child mortality, are due to the urbanization of settlements, the provision and extension of access to clean drinking water, the improvement of sanitary conditions, the extension and construction of sewage systems, the knowledge of the various infections and their spread, as well as the possibilities for their prevention, which is related to the limitation and / or suspension of a number of epidemics. Much later, the development of healthcare and medicine through the development of antibiotics and mass vaccination has been an important factor in demographic processes. As for the reasons listed above, during this phase of the demographic transition, the main trend of a significant increase in the birth rate over mortality is established, as a result of which a positive natural growth and continuous acceleration of the natural movement of the population with increasing volume of the new generations and consequently the formation of broad-based age pyramids. The next third phase is characterized by a decrease in the birth rate, the gradually decreasing inflow of new generations and thus the beginning of the aging of the population, and at the beginning of this phase the population still has a significantly younger age structure, demographic growth. The emergence of the third phase of the demographic transition can be sought on the one hand in the rapidly urbanizing populations and the intensive emancipation of women, which is why women today are often faced with the dilemma of choosing career careers or family and children. The increase in the share of women faced with this dilemma is also due to the expansion of education and the promotion of culture in the family planning process. After World War II, in many countries in Europe and North America, accelerated industrialization and markedly extensive development took place, which accelerated the process of attracting rural population to cities, which had an impact on internal migration towards a villagecity, accumulate huge urban agglomerations. Increasing productivity generates more income opportunities outside of the family and household, making choices between childbirth and childcare and income denial, which makes the birth rate more expensive and binds the process with parallel productivity gains. This process in part leads to new family prioritization not related to the number of children but to the possibility of providing appropriate education and the prospects for them. Here is the decline in the share of multi-family households that are formed by several families of successive generations and the establishment of single-family households and even informal families. As a result, the attitude towards extramarital births and lone parents is changing in the direction of alignment with other families. The views of the unfortunate marriage, which is not considered an irreparable, fatal error, also change. Gradually, there is a tendency to increase the frequency of cohabitations, resulting in a significant increase in the number of children from non-marital relationships. Of course cohabitation has certain characteristics, where two opposing social poles are distinctly observed, while the first cohabitation is carried out by ambitious, career-oriented parents with relatively high incomes, while in the second cohabitation it is motivated by economic uncertainty, the low material culture of individual weak social layers and social groups of society. In addition, there is a certain correlation between marriage and cohabitation with age, as young people are ready to marry often after completing their education, with age and 
income growth. The evolution of the relationship between man and woman and the transformation of families into a partnership between the two sexes is a consequence of different processes, both economic, sociological, historical, ethnic, religious, philosophical, political and others.

\section{IMPACT OF DEMOGRAPHIC PROCESSES ON SUSTAINABLE SOCIAL AND ECONOMIC DEVELOPMENT.}

The implementation of a particular demographic policy is linked to the creation and search for opportunities to achieve certain desired goals that affect the socio-economic development of the nation's and regions' beliefs. Demographic policy is linked to the implementation of measures to raise, promote or support birth rates or measures to reduce and reduce birth rates. As the impact of demographic processes on sustainable social and economic development can be traced in the following main directions:

- Reproduction of labor resources - number, age, gender, education

- In the case of employment, taking into account the different employment intensity by gender and age, and also by the number of children in the family, the intergenerational age gap, the educational and professional structure of migrants and emigrants

- In the case of unemployment, taking into account unemployment rates by gender, age groups, young people and persons in retirement age

- Taking into account the level of income by gender and age

- Reallocation of income depending on the size and composition of the family

- In shaping the poverty level. Increasing or decreasing the number of children in small and large families, respectively, affects the increase or decrease of the poverty level of different ethnic groups.

- Demand - the growth of the population leads to an increase in demand, and the relative growth of children influences the demand for children's goods. Changes in population size, marriage, and divisiveness, have led to a change in demand for housing (by squares and by number of rooms).

- Educational services. Growing or decreasing fertility rates over a period of time leads to a 1 or 3-year increase or decrease in demand for childcare facilities, 6 or 7 years of primary education, 13 or 14 years of secondary education, and 18 or 19 years of demand for higher education.

- Healthcare. The change in the number of the population alters the burden on healthcare establishments and the sex-age structure, the birth rate and the mortality rate, and the proportion of doctors in the specialties, children's polyclinics, maternity hospitals, etc.

- Social support, child-raising and child-raising allowances, development of the social service provision network of the population [1].

Businesses consider the demographic factor not only in terms of demand (as a factor for the formation of aggregate consumer demand and its territorial differentiation) but also in terms of supply (as a factor for employment formation). The availability of highly educated, skilled and unoccupied work resources is an important factor in reducing finance to prepare and stimulate immigration in a given workforce region. In this way, companies are open to discovering new productions in these regions without the need to invest in attracting or training high technology staff, for example. An important trend in the reproduction of the population, both in developed countries and in our country, is its aging. Aging populations affect the economy in several main directions: employment and 
quality structure of the workforce, including the employment of pensioners; pension provision and standard of living for older people as a significant proportion of the population; health, organization of healthcare and social services. The aging of the labor force influences its productivity in two main directions: in connection with the increase of the traineeship, the working habits, the experience and the qualification increase. In the sectors with a stable and traditional professional qualification structure, it plays a positive role. In the prospective branches of science and technology (information) revolution, secondary and tertiary education is of great importance, the ability to adapt quickly to the changing technical and technological conditions. These characteristics, however, are more inherent to young people. They are quick to master and use the achievements of science, they are more educated, more mobile, both professionally and territorially. At the same time, young people (up to 25 years) do not have the necessary professional experience and are insufficiently adaptable to the workforce. The labor force in Bulgaria has undergone a change with the aging of the population. The share of older workers is increasing. This requires a change in the labor market, education and health. [1] On the other hand, migratory movements are closely linked to urbanization, which leads to rapid growth of cities and the emergence of gigantic urban agglomerations, where urban agglomerations are becoming an attractive center for a further wave of immigrants. As a result, by 2050 , over $70 \%$ of the world's population is expected to live in cities. Typically, the rural population has always had a higher fertile capacity than the urban, but the birth rate in the villages is lower because of the mass migration of young people to the cities. In the future, there is a certain delay in marriage and a corresponding increase in the average age at formal marriages, as well as a relative decrease in their number at the expense of informal ones and the spread of fairly free forms of cohabitation, childbirth and childcare.

\section{PROSPECTS FOR DEMOGRAPHIC DEVELOPMENT OF THE POPULATION.}

The influence of the demographic determinants on the socio-economic development of the regions and countries is becoming ever more important, which is why the demand for and justification of explanations of the driving forces of the demographic processes in the modern world is becoming more and more current. In existing demographic projections, the world population is dealt with in two main groups - developed and developing countries, with the second group focusing on the least developed countries. Various combinations and variants of the likely future state of the population are presented. Worldwide forecasts are developed by various international organizations such as the World Bank, the International Labor Organization, the World Health Organization, EUROSTAT, the International Institute for Applied System Analysis, the Max Planck Institute for Demographic Studies, and many of the leading national statistical authorities and other government offices, as well as from a number of universities, consultancy companies and others. The forecasts are aimed at highlighting specific issues that are related to a number of issues such as trade and economic development, employment and unemployment, health, social security, environmental issues, etc. Also attention to the demographic processes in Europe and the future of its populations is also addressed in the bodies of the European Union. Demographic forecasts are made for individual areas, settlements that are related to the implementation of specific investment projects or townplanning solutions. On the basis of the forecast data, estimates are made for the future 
development of income and consumption of the population, the labor market, health, education, transport and other needs. An important factor in demographic projections are migratory movements, often based on the observed trends in the recent past, taking into account to a certain extent the policies of the states in terms of the mechanical movement of the population. It is often observed that statistical information on migratory movements is considerably inferior to that of natural movement of the population, as the registration of migration processes in different regions of the world, part of the trips remain outside administrative supervision and a certain part of the world's population, mostly in developing countries, does not lead a sedentary way of life. Also, the reliability of the data is also related to the lack of address or similar registration in some developed countries. Very often data from representative surveys are used to study migration, either in population censuses or in some single sociological surveys that shed light on individual aspects of the mechanical movement of the population. To date, demographic growth in most European countries is due primarily or even entirely to immigrants, as in the last twenty years, intensive settlements have been observed in Western Europe. Migration movements affect the location of the population, but also indirectly by modifying the size and age structure of the maternity contingents, as a result of which the intensity and even the direction of the natural movement may change. There is a change in the borderline between external and internal migration, while increasing and intensifying daily work trips, weekly and seasonal movements of the workforce, free movement within the European Union. The problem of actually taking into account the migration flows is the blurring of the concepts of residence, residence, permanent population, address registration, factual population, legal population, which necessitates rethinking the relevant classifiers and nomenclatures, as well as updating the criteria in assessing migratory movements or rather it is necessary to seek new approaches to studying these processes. The world population is expected to exceed 9 billion by the middle of the 21 st century but is expected to stabilize after around 2100 at levels of about 9 billion [2]. In the field of migration movements, demographic statistics are faced with a number of problems, particularly in the case of increased population mobility by the end of the 20th century, as registration of migration acts is usually incomplete, which makes it difficult to identify the difference between ordinary and relatively short journeys actual resettlements. There is also a frequent transformation of settlements in settlements that remain outside the scope of initial administrative surveillance. There are countries where internal migration as such is not observed, as there is no address registration (the Netherlands, USA) or the population register where all demographic events for individuals should be reflected and reconciled in the part about mechanical movement and the location of the population in the various regions of the country does not give satisfactory results (according to Swedish statisticians). [3] The territorial disposition of the human factor is definitely the main driving force in economic and social development, which determines the increase of the demographic data needs of the population inhabiting a territorial administrative unit. Because of this, the use of specialized representative surveys, marketing studies, and various drills is very often used to fill existing information gaps to support initiatives by both the public and the private sector, as it is extremely important to know the factual, the available population and its territorial location. As a result, demographic projections should be construed as being statistically built with characteristic features. Often in developing countries, the information base for the forecasts is also problematic for natural population movements, with even mortality and life expectancy estimates uncertain and often extracted indirectly with partial or total 
absence of relevant administrative reporting [3]. Demographics predict hypotheses about higher, even increasing, fertility rates for women, which is why higher birth rates are expected in the future, although many other conditionality related to the mortality and duration of life, migratory movements, the quality of the statistical material used, and so on.

\section{CONCLUSION}

The dynamics of world population population numbers is determined by the natural movement of the population, with a rapid increase in the absolute number of the population worldwide, which exacerbates the contradictions between rapid population growth, economic problems and natural constraints negative attitudes such as malnutrition and related chronic diseases, various forms of social opposition, environmental pollution and inefficient use of natural resources. In the interaction between demographic development and the economy, a number of parameters can be considered. First, employment rates are of central importance. Age dependency ratios are of limited significance as long as a large part of the working-age population is excluded from the labour market: the real issue is the number of economically active persons that can contribute to income generation to cover the needs of the non-economic population. Individual accumulation of capital is also a significant parameter: the economically inactive population will, to a varying degree, be able to cover its own needs depending on the capital it has accumulated and the revenue this capital generates. [4] The results show that there are countries in the various regions of the world who demonstrate good practices with regard to the use of information and communication technologies to provide services and to create citizens' ownership of the decision-making processes. An important trend in recent years has been the development of people-driven services, which simultaneously reflect the needs of people and have been suggested by them. On the other hand, disparities between countries also lie in the lack of access to technology, the high poverty and disadvantages of significant population groups in individual countries that we can relate to preventing people from benefiting from the benefits of information and communication technologies and management [5]. In our country, attempts have been made to curb the demographic decline of the nation, as the main strategic objective is to slow the pace of reduction in the number of the population with a view to achieving a trend of stabilization in the long term and of ensuring high quality of human capital[6] Particular attention should be paid to the demographic factor in the shaping of social and economic development programs and the implementation of regional economic and social policies.[7]

\section{REFERENCES}

[1] Naydenov, Kl., Ivanov, M., Annual of the University of mining and geology „St. Ivan Rilski“, Vol. 59, Part IV, Humanitarian sciences and Economics, 2016

[2]United Nations Population Division (2004), World Population in 2300 (ESA/P/WP.187/Rev.1/ 24 March 2004) available to http://www.un.org/esa/population/publications/longrange2/2004worldpop2300reportfinalc.pdf on 23.02.2018.

[3] Cholakov, N., DEMOGRAPHIC PROCESSES - HISTORY, TRENDS, PROSPECTS, available 
http://www.unwe.bg/uploads/ResearchPapers/Research\%20Papers_vol1_2012_No4_N\%20Cho lakov.pdf on 23.02.2018.

[4] The impact of demographic change on European regions, available to https://cor.europa.eu/en/documentation/studies/Documents/The\%20impact\%20of\%20de mographic\%20change\%20on\%20European\%20regions/Impact_demographic_change_e uropean_regions.pdf on 23.02.2018.

[5] Naydenov, Kl., Regional differences and trends in e-governance, IX-th International scientific conference „E-governance and E-communications“, 2017, p. 45-52, ISSN 2534-8523

[6] Naydenov, Kl., „The demographic deficit in the economic development of the Republic of Bulgaria - consequences and decisions“, SGEM 2017, Book 1 Modern Science Volume IV, p. 785 - 792, ISBN 978-619-7408-16-4

[7] Naydenov, Kl., Influence of the demographic factor on the regional social and economic development", SGEM 2017, Volume I, p.363 - 369,ISBN 978-619-7408-23-2 